\title{
Simultaneous determination of phytochemical constituents in Paeonia lactiflora extracts using the HPLC-UV method
}

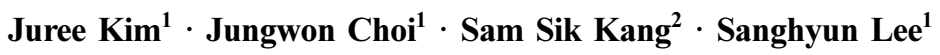 \\ Received: 16 December 2020 / Accepted: 28 December 2020 / Published Online: 31 March 2021 \\ (C) The Korean Society for Applied Biological Chemistry 2021
}

\begin{abstract}
Qantitative analysis of six compounds: (+)-catechin, benzoic acid, gallic acid methyl ester, paeonol, paeoniflorin, and albiflorin from Paeonia lactiflora extracts was performed using high-performance liquid chromatography and an ultraviolet (UV) detector, following different extraction methods. A reverse-phase column was used in a gradient elution system, and UV detection was performed at $280 \mathrm{~nm}$. The results showed that the quantity of paeoniflorin was the highest in ethanol and water extracts (73.89 and $57.87 \mathrm{mg} / \mathrm{g}$, respectively) among the six compounds. This study contributes a good analysis method for the contents of $P$. lactiflora and would be propitious for developing medicines and functional foods.
\end{abstract}

Keywords Albiflorin · Benzoic acid · (+)-Catechin · Gallic acid methyl ester ' Paeonia lactiflora ' Paeoniflorin 'Paeonol · Quantitative analysis

\section{Introduction}

Paeonia lactiflora (PL), belonging to the family Paeoniaceae, is a species of herbaceous perennial flowering plants, and its origin is in central and eastern Asia, from eastern Tibet, across northern

Sanghyun Lee $(\square)$

E-mail: slee@cau.ac.kr

${ }^{1}$ Department of Plant Science and Technology, Chung-Ang University, Anseong 17546, Republic of Korea

${ }^{2}$ Natural Products Research Institute and College of Pharmacy, Seoul National University, Seoul 08826, Republic of Korea

This is an Open Access article distributed under the terms of the Creative Commons Attribution Non-Commercial License (http://creativecommons. org/licenses/by-nc/3.0/) which permits unrestricted non-commercial use, distribution, and reproduction in any medium, provided the original work is properly cited.
China to eastern Siberia. In particular, the roots of PL were traditionally consumed as oriental medicine [1]. PL has been used to treat dysmenorrhea, amenorrhea, and spasm [2,3]. In addition, PL is well known for its vasodilatory [4], anti-hyperlipidemic [5], anti-oxidant, and anti-bacterial effects [6].

PL has a variety of bioactive components, such as monoterpenes $[7,8]$, triterpenes [9,10], volatile oils [11], tannins [12], stilbenes [13], flavonoids [14], and polyphenols [15,16]. Among them, paeoniflorin and albiflorin are the primary components of PL [17]. Paeoniflorin has been reported to have anti-inflammatory, immunomodulatory [18], spasmolytic [19], and hypoglycemic activities [20]. Albiflorin is effective for the treatment of inflammation [21], neuropathic pain [22], osteoporosis [23], and depression [24].

In this study, we aimed to quantify the phytochemical constituents in PL using a high-performance liquid chromatography (HPLC)ultraviolet (UV) detector and compare the quantity of each compound in the extract after different extraction methods.

\section{Materials and Methods}

\section{Plant materials}

The ethanolic (EtOH) (3-19-0091) and water (3-19-0048) extracts of PL were provided by Korea Institute of Oriental Medicine, Daejeon, Korea.

\section{Chemicals and apparatus}

Chromatographic analysis was performed using HPLC system (Agilent technology 1290 Infinity II, Santa Clara, CA, USA) equipped with a pump, an auto-sampler, and a UV detector. Solvents used for HPLC (water and acetonitrile) were HPLC grade and purchased from J. T. Baker (Avantor, Radnor, PA, USA). Acetic acid (99.7\%) was purchased from Samchun Pure Chemicals (Pyeongtaek, Korea). Six compounds: (+)-catechin, benzoic acid, gallic acid methyl ester, paeonol, paeoniflorin, and 
<smiles>Oc1cc(O)c2c(c1)O[C@H](c1ccc(O)c(O)c1)[C@H](O)C2</smiles>

1<smiles>O=C(O)c1ccccc1</smiles>

2

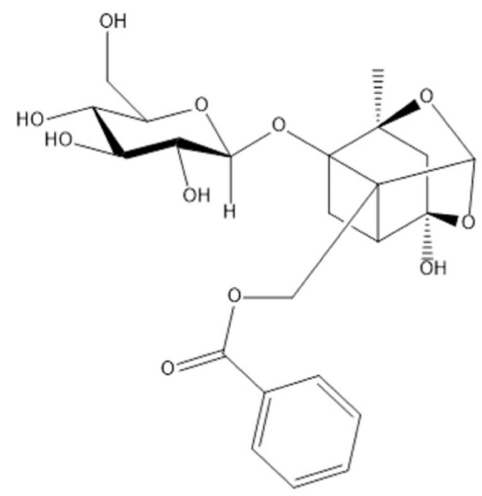

5<smiles>COC(=O)c1cc(O)c(O)c(O)c1</smiles>

3<smiles>COc1ccc(C(C)=O)c(O)c1</smiles>

4

Fig. 1 Chemical structures of the six compounds $[(+)$-catechin (1), benzoic acid (2), gallic acid methyl ester (3), paeonol (4), paeoniflorin (5), and albiflorin (6)] in PL

albiflorin (Fig. 1) were obtained from Natural Product Institute of Science and Technology (www.nist.re.kr), Anseong, Korea.

\section{Sample extraction methods}

Ethanolic [70\% EtOH (4 L)] extraction of dried and crushed PL $(1 \mathrm{~kg})$ was performed under sonication for $1 \mathrm{~h}$; the extraction process was run two times. Subsequently, the samples were filtered, evaporated at $37^{\circ} \mathrm{C}$, freeze-dried, and homogenized using a $600-\mu \mathrm{m}$ sieve to obtain EtOH extract of PL (EEP). Water (4 L) extraction of dried and crushed PL (1 kg) was performed for $3 \mathrm{~h}$ under reflux conditions $\left(100^{\circ} \mathrm{C}\right)$. After extraction, the samples were filtered using a $53-\mu \mathrm{m}$ sieve, evaporated at $37^{\circ} \mathrm{C}$, and homogenized using a $600-\mu \mathrm{m}$ sieve to obtain water extract of PL (WEP). The homogenized powders were stored in a tight-sealed bottle and kept in a refrigerator away from light until analysis.

\section{Preparation of samples for HPLC}

EEP and WEP (1 mg each) were dissolved separately in methanol $(\mathrm{MeOH})$ under sonication for $20 \mathrm{~min}$ and filtered using a PVDF Membrane filter of $0.45-\mu \mathrm{m}$ pore size. These were used as the experimental stock solutions. One milligram of each of the six compounds was dissolved seperately in $\mathrm{MeOH}$ under sonication for $20 \mathrm{~min}$ and filtered using a $0.45-\mu \mathrm{m}$ PVDF membrane filter. These were used as the standard solutions.

\section{HPLC conditions}

Quantitative analyses were performed using a reverse-phase HPLC system with an INNO C18 column $(250 \mathrm{~mm} \times 4.6 \mathrm{~mm}$, $5 \mu \mathrm{m})$. UV detection was at $280 \mathrm{~nm}$, and the temperature of the column was maintained at room temperature. The injection volume was $10 \mu \mathrm{L}$, and the flow rate was set to $1 \mathrm{~mL} / \mathrm{min}$. The 
Table 1 Data from the calibration curves of the six compounds [(+)-catechin (1), benzoic acid (2), gallic acid methyl ester (3), paeonol (4), paeoniflorin (5), and albiflorin (6)]

\begin{tabular}{cccc}
\hline \hline Compound & $\mathrm{t}_{\mathrm{R}}$ & Calibration equation $^{a}$ & ${\text { Correlation coefficient, } r^{2 b}}^{2 b}$ \\
\hline $\mathbf{1}$ & 16.313 & $\mathrm{Y}=3.2366 \mathrm{X}+39.55$ & 0.9989 \\
$\mathbf{6}$ & 16.525 & $\mathrm{Y}=37.871 \mathrm{X}+27.792$ & 0.9992 \\
$\mathbf{5}$ & 20.473 & $\mathrm{Y}=0.6301 \mathrm{X}+44.929$ & 0.9987 \\
$\mathbf{2}$ & 22.099 & $\mathrm{Y}=0.8803 \mathrm{X}+8.6333$ & 0.9999 \\
$\mathbf{4}$ & 30.719 & $\mathrm{Y}=4.8757 \mathrm{X}+105.49$ & 0.9996 \\
\hline
\end{tabular}

${ }^{a} \mathrm{Y}=$ peak area, $\mathrm{X}=$ concentration of the standards $(\mathrm{mg} / \mathrm{mL})$

${ }^{b} r^{2}=$ correlation coefficient based on five data points in the calibration curves

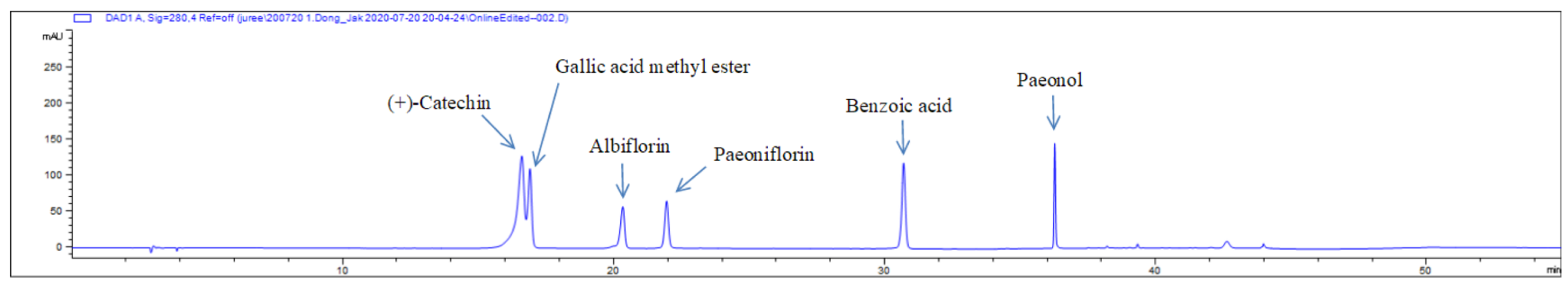

Fig. 2 HPLC chromatogram of the six compounds

mobile phase of the gradient elution system consisted of $0.5 \%$ acetic acid in water (A) and acetonitrile (B). The composition of the gradient elution system was as follows: $95 \% \mathrm{~A}$ at $0 \mathrm{~min}, 75 \%$ $\mathrm{A}$ at $25 \mathrm{~min}, 60 \% \mathrm{~A}$ at $30 \mathrm{~min}, 100 \% \mathrm{~B}$ at $35 \mathrm{~min}, 100 \% \mathrm{~B}$ at 40 $\min , 95 \% \mathrm{~A}$ at $45 \mathrm{~min}$, and $95 \% \mathrm{~A}$ at $55 \mathrm{~min}$.

\section{Calibration curves}

The standard stock solutions were prepared by dissolving the compounds in $\mathrm{MeOH}(1 \mathrm{mg} / \mathrm{mL})$. The working solutions, which were prepared by serially diluting the stock solutions, were used to construct the calibration curves. The calibration functions of the standards were calculated using the peak area $(\mathrm{Y})$, concentration $(\mathrm{X}, \mathrm{mg} / \mathrm{mL})$, and mean \pm standard deviation $(\mathrm{n}=3)$.

\section{Results and Discussion}

PL has anti-oxidant [25], anti-diabetic [26], anti-microbial [27], and anti-hyperlipidemic effects [28]. A recent study revealed the skin-depigmenting potential of PL in hyperpigmentation disorders [29]. It has a variety of bioactive components, such as paeoniflorin, albiflorin, benzoic acid, oleanolic acid, hederagenin, and oxypaeoniflorin [16]. Among them, paeoniflorin and albiflorin are the primary constituents of PL [17].

Quantitative analysis of the six representative compounds in EEP and WEP was performed using HPLC with a reverse phase column and gradient elution of solvents $\mathrm{A}$ and $\mathrm{B}$ in the mobile phase. The HPLC method showed good separation, and a wavelength of $280 \mathrm{~nm}$ was found to be effective for detection. The data from the calibration curves of the standards are shown in
Table 1. The calibration curves were constructed by linearly plotting the peak area against the prepared concentrations of the standard solutions and were analyzed using linear regression. The linear regression coefficients $\left(r^{2}\right)$ for the standards were between 0.9987 and 0.9999 .

Chromatograms of the standard solutions of the six compounds are shown in Fig. 2. Chromatographic peaks of $(+)$-catechin, gallic acid methyl ester, albiflorin, paeoniflorin, benzoic acid, and paeonol showed good separation in EEP and WEP (Fig. 3). Table 2 shows the quantity of the six compounds in EEP and WEP. The results showed that the quantity of the six compounds was generally higher in EEP than in WEP. However, considering the dry weights, the quantity of albiflorin, paeoniflorin, and benzoic acid was higher in WEP than in EEP. This seems to be due to the variation in yield according to the extraction method. The extraction yields of EEP and WEP were $16.63 \%(\mathrm{w} / \mathrm{w})$ and $27.64 \%(\mathrm{w} / \mathrm{w})$, respectively, which shows the extraction efficiency of water extraction method. Among the six compounds in PL, the quantity of paeoniflorin was remarkably high, followed by albiflorin, in both EEP and WEP.

Choung et al. found suitable conditions for analysis of paeoniflorin using different extraction methods and times [30]. They reported that reflux extraction produced sufficient yield in $1 \mathrm{~h}$, whereas sonication extraction for 1 to $2 \mathrm{~h}$ yielded less quantity than reflux extraction. Additionally, there was no marked difference in yield even after 3 to $4 \mathrm{~h}$ of sonication extraction. Moreover, Kim et al. reported that, extraction using 70\% EtOH was effective when extracting from dried powder of PL [31]. Another study showed that paeoniflorin was efficiently extracted with $70 \% \mathrm{MeOH}$ or water [32], although not with pure EtOH or 


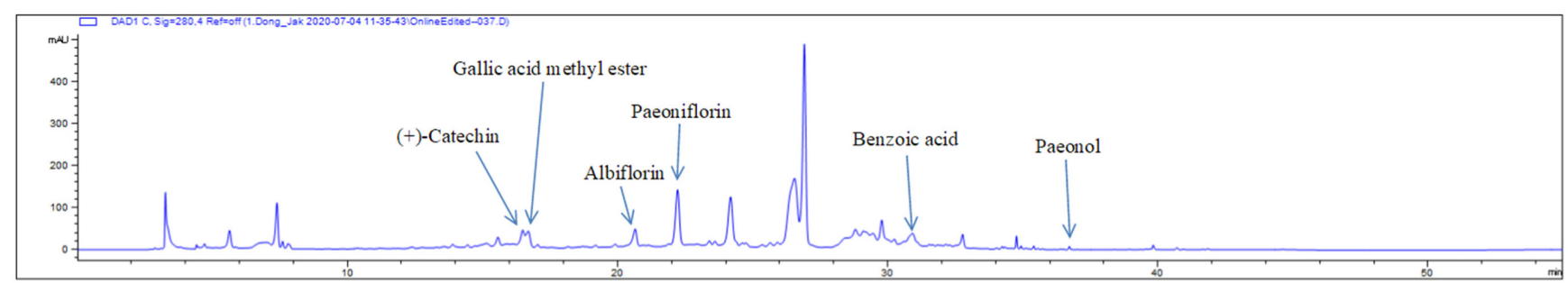

(A)

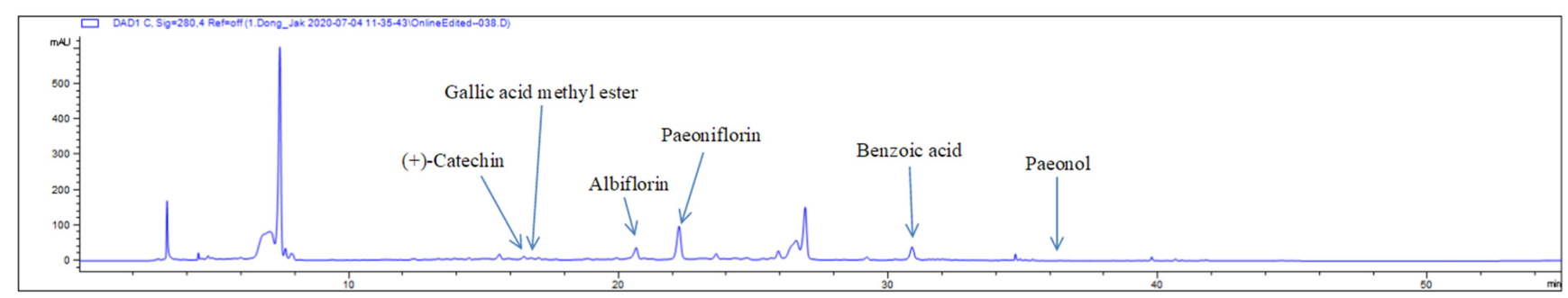

(B)

Fig. 3 HPLC chromatograms of EEP (A) and WEP (B)

Table 2 Quantity of the six compounds [(+)-catechin (1), benzoic acid (2), gallic acid methyl ester (3), paeonol (4), paeoniflorin (5), and albiflorin (6)] in the EEP and WEP

\begin{tabular}{cccccc}
\hline \hline \multirow{2}{*}{ Compound } & \multicolumn{3}{c}{ EEP } & & \multicolumn{2}{c}{ WEP } \\
\cline { 2 - 3 } \cline { 5 - 6 } & $(\mathrm{mg} / \mathrm{g}$ ext. $)$ & $(\mathrm{mg} / \mathrm{g} \mathrm{DW})$ & & $(\mathrm{mg} / \mathrm{g}$ ext. $)$ & $(\mathrm{mg} / \mathrm{g} \mathrm{DW})$ \\
\hline $\mathbf{1}$ & $1.58 \pm 0.01$ & $0.26 \pm 0.00$ & & $0.37 \pm 0.05$ & $0.10 \pm 0.01$ \\
$\mathbf{3}$ & $0.21 \pm 0.01$ & $0.03 \pm 0.00$ & & trace & trace \\
$\mathbf{6}$ & $30.03 \pm 0.21$ & $4.98 \pm 0.03$ & & $23.14 \pm 0.17$ & $6.39 \pm 0.05$ \\
$\mathbf{5}$ & $73.89 \pm 0.76$ & $12.27 \pm 0.13$ & & $57.87 \pm 0.20$ & $15.97 \pm 0.06$ \\
$\mathbf{2}$ & $3.45 \pm 0.10$ & $0.57 \pm 0.02$ & & $2.96 \pm 0.02$ & $0.82 \pm 0.01$ \\
$\mathbf{4}$ & $0.02 \pm 0.00$ & trace & trace & trace \\
\hline
\end{tabular}

$\mathrm{MeOH}$ [33]. In this study, the quantity of paeoniflorin was higher in EEP than in WEP, which shows that the extraction using $70 \%$ EtOH is more suitable. Additionally, if reflux extraction had been used for the ethanolic extracion of PL, the extraction yield would have increased.

Paeoniflorin has anti-hyperlipidemic [28], anti-wrinkle [34], and anti-depressant effects [35]. Albiflorin has anti-inflammatory [36], hematopoietic [37], and anti-depressant [38] effects. We quantified a total of six phytochemical constituents of PL, including albiflorin and paeoniflorin, the major components of PL, using different extraction methods. In conclusion, this study provides a good analysis method for determining the contents of PL. These results would be beneficial in developing medicines and functional foods.

Acknowledgments We thank the Korean Herbarium of Standard Herbal Resources (EEP, 3-19-0091; WEP, 3-19-0048) and Dr. Jin-Mu Yi, Korea Institute of Oriental Medicine, Daejeon 34054, Republic of Korea, for the provision of materials.

\section{References}

1. Lee TB (1985) Colored Flora of Korea I. Hyangmoon Press, Seoul

2. Zhang W, Dai S (2012) Mechanisms involved in the therapeutic effects of Paeonia lactiflora Pallas in rheumatoid arthritis. Int Immunopharmacol 14: 27-31

3. Wu SH, Wu DG, Chen YW (2010) Chemical constituents and bioactivities of plants from the genus Paeonia. Chem Biodivers 7: 90104

4. Goto H, Shimada Y, Akechi Y, Kohta K, Hattori M, Terasawa K (1996) Endothelium-dependent vasodilator effect of extract prepared from the roots of Paeonia lactiflora on isolated rat aorta. Planta Med 62: 436-439

5. Li J, Chen CX, Shen YH (2011) Effects of total glucosides from paeony (Paeonia lactiflora Pall) roots on experimental atherosclerosis in rats. J Ethnopharmacol 135: 469-475

6. Zhou JX, Braun MS, Wetterauer P, Wetterauer B, Wink M (2019) Antioxidant, cytotoxic, and antimicrobial activities of Glycyrrhiza glabra L., Paeonia lactiflora Pall., and Eriobotrya japonica (Thunb.) Lindl. extracts. Medicines (Basel) 6: 43

7. Braca A, Kiem PV, Yen PH, Nhiem NX, Quang TH, Cuong NX, Minh CV (2008) New monoterpene glycosides from Paeonia lactiflora. Fitoterapia 79: 117-120 
8. Wang HB, Gu WF, Chu WJ, Zhang S, Tang XC, Qin GW (2009) Monoterpene glucosides from Paeonia lactiflora. J Nat Prod 72: 13211324

9. Kamiya K. Yoshioka K, Saiki Y, Ikuta A, Satake T (1997) Triterpenoids and flavonoids from Paeonia lactiflora. Phytochemistry 44: 141-144

10. Ikuta A, Kamiya K, Satake T, Saiki Y (1995) Triterpenoids from callus tissue cultures of Paeonia species. Phytochemistry 38: 1203-1207

11. Kumar N, Motto MG (1985) Volatile constituents of peony flowers. Phytochemistry 25: 250-253

12. Tanaka T, Fukumori M, Ochi T, Kouno I (2003) Paeonianins A-E, new dimeric and monomeric ellagitannins from the fruits of Paeonia lactiflora. J Nat Prod 66: 759-763

13. Kim HJ, Chang EJ, Bae SJ, Shim SM, Park HD, Rhee CH, Park JH, Choi SW (2002) Cytotoxic and antimutagenic stilbenes from seeds of Paeonia lactiflora. Arch Pharm Res 25: 293-299

14. Jia N, Shu QY, Wang LS, Du H, Xu YJ, Liu ZA (2008) Analysis of petal anthocyanins to investigate coloration mechanism in herbaceous peony cultivars. Sci Hortic 117: 167-173

15. Guo D, Ye G, Guo H (2006) A new phenolic glycoside from Paeonia lactiflora. Fitoterapia 77: 613-614

16. Kang SS, Kim JS, Yun-Choi HS, Han BH (1993) Phytochemical studies on Paeonia radix. Kor J Pharmacogn 24: 247-250

17. Choung MG, Kang KH (1997) Isolation and determination of paeoniflorin and albiflorin in Korea peony (Paeonia lactiflora Pall) root. Korean J Medicinal Crop Sci 5: 249-254

18. He DY, Dai SM (2011) Anti-inflammatory and immunomodulatory effects of Paeonia lactiflora Pall., a traditional Chinese herbal medicine. Front Pharmacol 2: 10

19. Abdel-Hafez AA, Meselhy MR, Nakamura N, Hattori M, Watanabe H, Murakami Y, El-Gendy MA, Mahfouz NM, Mohamed TA (1999) Anticonvulsant activity of paeonimetabolin-I adducts obtained by incubation of paeoniflorin and thiol compounds with Lactobacillus brevis. Biol Pharm Bull 22: 491-197

20. Hsu FL, Lai CW, Cheng JT (1997) Antihyperglycemic effects of paeoniflorin and 8-debenzoylpaeoniflorin, glucosides from the root of Paeonia lactiflora. Planta Med 63: 323-325

21. Wang QS, Gao T, Cui YL, Gao LN, Jiang HL (2014) Comparative studies of paeoniflorin and albiflorin from Paeonia lactiflora on antiinflammatory activities. Pharm Biol 52: 1189-1195

22. Zhou J, Wang L, Wang J, Wang C, Yang Z, Wang C, Zhu Y, Zhang J (2016) Paeoniflorin and albiflorin attenuate neuropathic pain via MAPK pathway in chronic constriction injury rats. Evid Based Complement Alternat Med 2016: Article ID 8082753 (11 pages)

23. Suh KS, Choi EM, Lee YS, Kim YS (2013) Protective effect of albiflorin against oxidative-stress-mediated toxicity in osteoblast-like
MC3T3-E1 cells. Fitoterapia 89: 33-41

24. Wang YL, Wang JX, Hu XX, Chen L, Qiu ZK, Zhao N, Yu ZD, Sun SZ, Xu YY, Guo Y, Liu C, Zhang YZ, Li YF, Yu CX (2016) Antidepressantlike effects of albiflorin extracted from Radix Paeoniae Alba. J Ethnopharmacol 179: 9-15

25. Heo JI, Kim JH, Lee JM, Kim SC, Park JB, Kim J, Lee JY (2013) Antioxidant activity and its mechanism of Paeonia lactiflora Pall extract. Nat Prod Sci 19: 49-53

26. Lee S, Ji S (2004) Effect of Paeonia lactiflora extracts on $\alpha$-glucosidase. Nat Prod Sci 10: 223-227

27. Park KD, Cho SH (2010) Antimicrobial characteristics of Paeonia lactiflora Pall. extract tested against food-putrefactive microorganisms. Korean J Food Preserv 17: 706-711

28. Yang HO, Ko WK, Kim JY, Ro HS (2004) Paeoniflorin: an antihyperlipidemic agent from Paeonia lactiflora. Fitoterapia 75: 45-49

29. Qiu J, Chen M, Liu J, Huang X, Chen J, Zhou L, Ma J, Sextius P, Pena AM, Cai Z, Jeulin S (2016) The skin-depigmenting potential of Paeonia lactiflora root extract and paeoniflorin: in vitro evaluation using reconstructed pigmented human epidermis. Int J Cosmet Sci 38: 444 451

30. Choung MG, Kang KH (1994) Extraction methods and HPLC analysis conditions of paeoniflorin in peony, Paeonia lactiflora Pall. Korean J Crop Sci 39: 542-547

31. Kim TK, Kim KJ, Joo GJ, Rhee IK (1997) Changes of paeoniflorin content in peony roots by heat-treatmnt. Korean J Food Preserv 4: 69-75

32. Kim TK, Joo GJ, Chung JD, Rhee IK (1996) Analysis of the content of paeoniflorin in peony roots cultivated on kyeongbuk area. Agric Res Bull Kyungpook Natl Univ 14: 15-28

33. Asakawa N, Hattori T, Ueyama M, Shinoda A, Miyake Y (1979) Determination of paeoniflorin in Paeony extract by high performance liquid chromatography. Yakugaku Zasshi 99: 598-601

34. Cho WG, Kyung KY, Yu SM (2009) Stability of paeoniflorin used as anti-wrinkle agents in emulsions. J Korean Oil Chem Soc 26: 191-198

35. Qiu F, Zhong X, Mao Q, Huang Z (2013) The antidepressant-like effects of paeoniflorin in mouse models. Exp Ther Med 5: 1113-1116

36. Xu X, Liu H, Pan Y, Liu Z, Chen D, Zhang L, Yuang H (2020) Albiflorin attenuates inflammation and apoptosis by upregulating AMPK-mediated expression of CDX2 in a mouse model of ulcerative colitis. Trop J Pharm Res 19: 995-999

37. Zhu Y, Zhou J, Wang L, Yang Z, Zhang J (2015) Hematopoietic effects of paeoniflorin and albiflorin on radiotherapy and chemotherapy-induced anemia mice. Planta Med 81: 928

38. Wang J, Wan Y, Zheng N (2020) Albiflorin ameliorates depressive-like behaviors in mice induced by chronic unpredictable mild stress. Curr Topics Nutraceut Res 18: 102-107 